\title{
Northern Labyrinth-A Key to Time, Space, Information
}

\author{
Alina Paranina \\ Herzen State Pedagogical University of Russia, St-Petersburg, Russia. \\ Email: galina_paranina@mail.ru \\ Received January $15^{\text {th }}, 2013$; revised May $28^{\text {th }}, 2013$; accepted June $19^{\text {th }}, 2013$ \\ Copyright (C) 2013 Alina Paranina. This is an open access article distributed under the Creative Commons Attribution License, which \\ permits unrestricted use, distribution, and reproduction in any medium, provided the original work is properly cited.
}

\begin{abstract}
Northern labyrinths are stone constructions of different shapes, dating back to III-I century BC. They mainly appear on capes on peninsulas and islands up to 13 - 25 metres above the sea level. Their area begins from Scilly Isles (England) and in the White Sea (Russia). Opinions of scientists concerning their designation are controversial. Assumptions about their practical meaning haven't been proved: no burials have been found underneath; fishing equipment cannot be located so far from the area of tides. Most of the scientists link labyrinths with the sphere of spiritual culture: sanctuaries, altars, sacred places, schemes of rituals and magical centers. Assumptions about calendar designation link the picture of labyrinth with schemes of orbits of the Sun, the Moon, planets and stars. However they do not explain the application of this technology. The author's concept is based on the opposite astronomical alignment by the shade of gnomon and considers peculiarities of the geographical space of Northern regions (polar days and white nights). The aim of the research is to prove that the key to decoding the picture of a classic Northern labyrinth is a gnomon located in the centre of the construction. Field research has been conducted on Zayztskiy Island in Solovetskiy Archipelago. Experiments of imitation modeling have been done, analysis of linguistic and toponymic materials, archeological, astronomical, ethnographical and mythological and other sources has been implemented. The research has proved that observations of the shade can help to create a calendar in a shape of a bispiral labyrinth. The structure of labyrinth is convenient for defining the North, dividing daytime and defining geographical altitude of the observation point. Orientation in space and time has become the basis for navigation frame of the territory. A set of stone instruments is the key of a navigation frame of the territory. Life-essential stages of the yearly lighting are reflected in specific geometry shapes of shades. They have been preserved in mythological images of the Sun, solar symbols and modern sign systems. Languages, alphabets, numeric systems can be considered as models of geographical space and have evolved thanks to observations of the light movement. Key results of the research: 1) in the past the Sun used to play the leading role in navigation in the North because reference points of the dark sky were not available for observation; 2) Northern labyrinths have been created and used thanks to gnomon; 3) gnomon has been in the sphere of sacred knowledge for a long time, it was integrated as sundial 2.5 thousand years ago; 4) most symbols of the Sun and the Time are connected with gnomon and labyrinth; 5) the Sun, observation tools and results of observations are reflected in models of the world around —navigation, linguistic, toponymical, mythological, sacral and semiotic; 6) The universal sense of Time helps to understand a wide range of meanings of a sign of a labyrinth: birth; life stages (status, transitions initiations); space time order; reproduction of the benefits, fertility, abundance; knowledge and forecast; management: law and order; eternity-revival; 7) Use of solar navigation in formation of information systems is based on steady ratios "natural process—a geometrical sign (the sum of shadows in one day or year)", universal opportunities of existential parameters for designation of objects, general availability and vital need of this knowledge.
\end{abstract}

Keywords: Northern Labyrinth; Gnomon; Sundial; Compass; Calendar; Information; World Models; Sign Systems

\section{Introduction}

Northern labyrinths are stone constructions in Northern Europe, one of their secrets is their complete identity to images on ancient coins from Crete in the Mediterranean sea (Figure 1). According to its definition, a labyrinththe house of labrys - an axe with two blades, which be- longed in different times to an Egyptian goddess of darkness and chaos Neit, a titanide Ree and to the god of light -Zeus.

The proposed concept of a labyrinth-gnomon-an orientation tool in space and time-conveys possible usage of a bispiral labyrinth as a sundial, compass and calendar. The shadow of the vertically adjusted object in the centre 


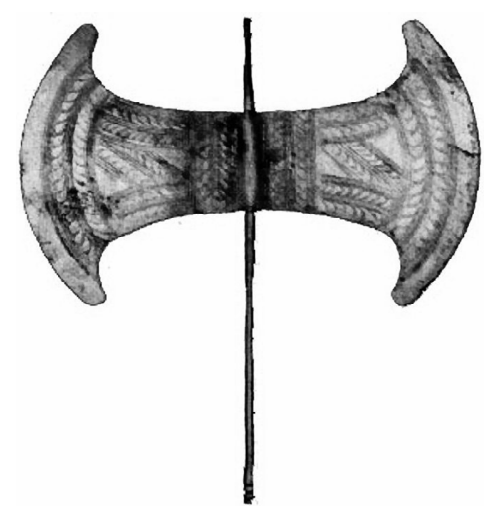

(a)

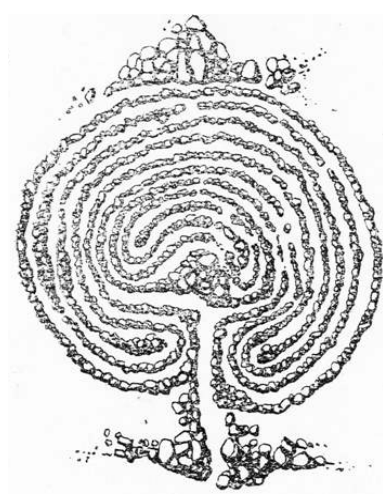

(b)

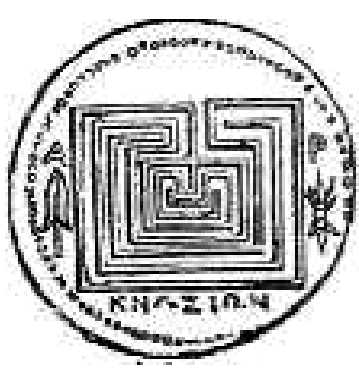

(c)
Figure 1. (a) A Knoss Labrys [3]; (b) Labrynth No. 1 of Bolshoy Zayatsky Island (radius = 5 meters) [4]; (c) A silver Knoss coin [3].

-a gnomon (from Greek-indicator) reflects the daily and annual movement of the Sun. Aggregate daily shadow has the shape of the fork and reminds horns or wings. Calendar changes in the geometry of shadows conform to the structure of the figure of the labyrinth and can be used to refer to the seasons [1,2].

Orientation with the gnomon opens deep layers of the content of myths and legends. We give examples of the new-navigation-reading of known stories: "the apple of knowledge" (coiled-coil design of the labyrinth-like an apple in the calendar section, see picture 1) provides a measure of time, depriving the illusion of eternity, while a wise snake, coiled-coil around the trunk-another copy of the labyrinth-gnomon. Spear and rope, shown next to the labyrinth at Knossos coins are the necessary and sufficient materials, tools for orientation in space-time (a spear is a gnomon, a rope is a universal basis for the proposed lines). Note that certain stories do not say, but do not distort the latent content they related to time, acting equivalent of higher learning, as in earlier versions have a more direct indication of the gnomon and light. So, in an ancient legend about the creation of the world, God cuts through the two-way road with an ax to light in the center of the labyrinth on one side Labrys pierces the gloom, the other-a (shadow appears) and discovers in his hand a fire torch instead of an ordinary ax.

The concept of the labyrinth-gnomon can uncover navigational basis of different models of the world and the existing sign systems, orientation in space-time gives simple answers to many problematic issues that have accumulated in archeology, history, philology, linguistics, toponymy, sphragistics and semiotics. One can suggest that preserved the ancient labyrinth of Egypt and its reduced copy on Crete, known only by historical evidence, is a solar observatory, in which the pointers are of different types of gnomon, among them-sunshine (reflected light) and direct beam. To test this hypothesis is necessary to study the ruins astroarcheological Egyptian labyrinth and palace of Knossos.

\section{Objects and Region of Research}

During 2009-2010, field and landscape astro-archeological research was undertaken on the White Sea islands: Bolshoy Zayatsky Island, Solovetsky Archipelago, Oleshin Island on the archipelago of Kuzov. A labyrinth in the center of the Russian plain, the high native river Don in its middle reaches, was explored as well. All elements of labyrinths, the gnomon shadow set in center of the labyrinth and nearby areas were researched, an assessment of landscape structure and topography was made.

For the processing of the material landscape-geographic and system-approach were used, astro-archeological methods and mathematical modeling with application basic trigonometric identities were applied as well. Results of Excel calculations: the geometry of the gnomon shadow for the year of its height and of the position in the maze for 9000 years, taking into account the precession of the equinoxes.

\section{Results}

Algorithm for astronomical observations in the northdimensional labyrinth has been defined. The resulting graph of the annual course of the gnomon shadow compared with the available historical evidence, legends and myths, are included in the complex analysis using linguistic, toponymy and ethnographic-ray material. Their importance was revealed as the basis for known characters and images.

Analysis of the annual dynamics of the midday shadow labyrinth number 1 of Bolshoy Zayatsky Island showed that it moves as uneven as a daily (Figures 2 and 3). For this reason, the division of rhythmically arranged arcs in equal parts is not feasible (note that unequal in length and were part of the day, highlighted by the sundial to improve them by tilting the gnomon on the North Star). At the same time, the main climax of the astronomical calendar in the arcs of the labyrinth is reflected 


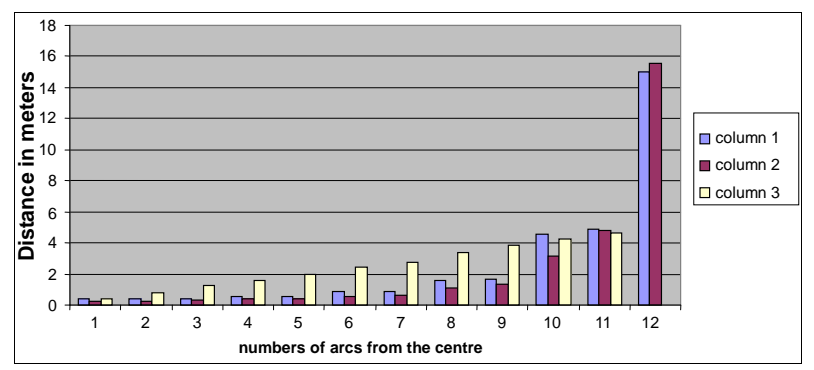

Figure 2. Correlation between radius $(\mathrm{m})$ of labyrinth arcs (column 3) and the yearly changes in vertical gnomon shadow lengths 5000 years ago (column 2) and in 2010 (column 1).

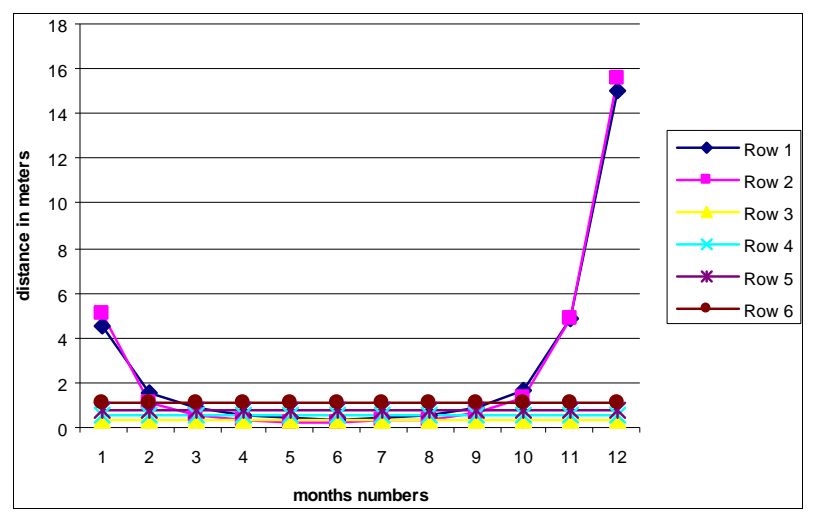

(a)

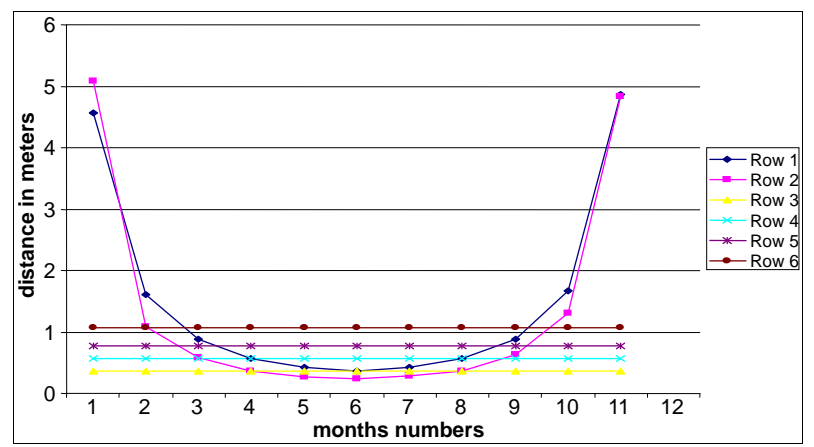

(b)

Figure 3. Shadow length and borders of the first two arcs: (a) for 12 months; (b) for 11 months (to enlarge scale). Row1 -according to the data of 2010, row-2-according to the data of 5000 years ago.

(Figure 3(b)): the first from the center of the arc corresponds to the short shadows of the summer solstice (next to it are the shadows all the summer months, which is consistent with the title this season in many European languages), the second arc corresponds to the equinoxes (separating the two main seasons, the first of which-the summer, and the second winter), the last, eleventh arc corresponds to a time, close to the winter solstice. Twelfth of the arc at a latitude close to the Arctic Circle was not used, because noon shadow in the days of the winter solstice was far removed from the center of the maze (Figure 2), for its observation is more suitable vertical screen of the stones-the northern addition (Figure 1).

In more southern regions where winter sun does not descend so low, and shadow is shorter, the extreme edge corresponds to the winter solstice, and the total number of arcs 12 and 7-in the classical forms of rational five arcs can be used twice. Schedule of the annual dynamics of the shadow (Figure 3(a)) shows a significant difference between the three winter months (1,11 and 12) February (2) and October (10) are transitional in nature, others form a smooth arc-in general, such a graphic account may be associated with as the solar boat, and the sequence of several such "Rooks" with the image of the wave. Note the increased contrast winter and summer in ancient times (Figure 3(a), row 2), the objectivity of the separation of warm half of the year (spring, summer and autumn, together account for seven months) in two phases - before and after the summer solstice.

Irregularity of the annual dynamics of the midday shadow has important consequences: 1) using the cycle of the moon and 2) the need for direct viewing-monitoring and fixing the azimuth of sunrise/sunset, 3) Consideration of the zodiac.

To count the days and moon phases in the structure of the labyrinth, you can use the four points of the ends of helices, between which, taken in pairs, there are 7 elements (the point ends of helices and in between the arc). Only between the two central points of all 5, but among them the central addition, the gnomon, which may "cost" 2 - 3 elements is not accidental in its portrayal of the upper part has a dichotomous branching (branch in the hands of Apollo, seated on omphalitis) and a trident or a crosshair (a sign of the Rurik). Alternation 7 - 8-dayquarters, and 28 - 29-day months, it is convenient to align the fractional duration of the phases of the moon. Thus, when using the lunar cycle, arcs, and other parts of the labyrinth could serve as elements of the counting. Sighting azimuth of sunrise/sunset could be in any form - direct or inverse.

Probably used a combination of both forms - as a tribute to tradition and system of duplication, verification, because the weather is not always conducive to observation of the shadow. Analysis of the conditions of the gnomon at different latitudes showed that the astronomical sensitive areas-the border zone coverage coincide with comfortable climatically areas of high atmospheric pressure, for which the characteristic of a large number of sunny days per year. These regions of the planet are known monuments of cultural heritage associated with the solar cult: Egypt, Mexico, Peru and others. Strong Siberian anticyclone in the heart of Eurasia was based curve assumptions about the development of solar worship in the territories now belonging to the natural habitat 
"lunar" cultural preferences. Megalithic monuments and Okunev culture in Khakassia, place names and ancient rock art solar bulls, circles, spirals and concentric forms provide a wealth of material to support this idea.

Regional analysis of size: the diameter of the gnomon and the site of observation midday shadows it possible to distinguish types of solar observatories: the labyrinth, staff, wand, ankh, and others. It is considered as special conditions for recording calendar information by using the gnomon in tropical regions.

Astronomical dating opportunities are shown in Figure 4. Astronomical parameters incorporated into the structure of images associated with the dynamics of the south elevation of the Sun above the horizon and the displacement of sunrise/sunset, depending on the position of the Earth's axis. Changing the tilt of the Earth axis of $21^{\circ} 55^{\prime}$ to $24^{\circ} 20^{\prime}$ with a period of 41,000 years, shifting the tropics and polar circles, which is reflected primarily on the points sunset/sunrise. Several thousand years ago, the slope was more modern, equal to $23^{\circ} 27^{\prime}$, and the Arctic Circle is located closer to the Bolshoy Zayatsky in the White Sea.

On the interval Meridian $1^{\circ} 33^{\prime}$ separating the Bolshoy Zayatsky Island from the polar circle azimuth points in the solstice sunrise varies by $20^{\circ}$. For 5000 years the azimuth of the susceptibility (number 1 ) at the latitude of the island has changed to $4.58^{\circ}$, and the midday height (number 2) only to $0.65^{\circ}$ (Figure 4). Consequently, benchmarks Back Sight of the gnomon are more stable than the direct guidance of sight, and serve as a guarantee of long-term use, use the calendar. A more dynamic azimuth of sunrise at the solstices is more effective as a basis for dating. The proposed structure-astronomical approach to the assessment of the likely time of construction of mazes based on the account a few factors, each of which brings its own limitations. First, significant differences in the definition of the point of sunrise: the emergence of a "whole disc", "his half of" or "first beam". If the first beam until the full disc is 13 minutes, then when $\mathrm{d}$ sun disc $0.5^{\circ}$ difference in azimuth of sunrise was respectively $1.47^{\circ}$ and $2.94^{\circ}$, which is equivalent to about its rejection in 1500 and 3000 . Another important factor-the duration of the allotted period of "no time" - the length of the shadows, beyond the line (outermost arc of the maze). For the ancient observatory trajectories of the North during this period is 1 - 2 months (from November to January for our time, from December to February, $5000 \mathrm{l}$. Ago), there are variants associated with a shorter period of "no time" or using the lunar cycle with 13 months of the year. Two other factors that limit the time of the labyrinth-calendar associated with the height of the gnomon and its location in central addition. If you define the universe of small height gnomon

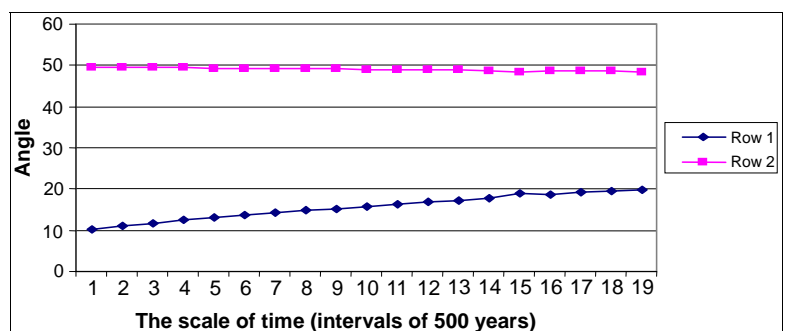

Figure 4. Midday height (line 1) and zenith of sunrise (line 2) at the summer solstice at latitude $65^{\circ} \mathrm{N}$ for 9000 years.

height of the arcs of the labyrinth may be irresistible for its shadow. To clarify the conditions in the next field season will be made by detailed measurements of the heights of all parts of the maze. Position in the center, determined by calculation, for 5000 years has shifted by $10 \mathrm{~cm}$ and if the trend persists, reserves not enough space for more than 2500 years. The last restriction on focusing imposes age relief, clarification of which range from 7000 to 9000 years is underway geomorphology. Our tentative estimate of the age of the labyrinth 7500 - 8000 years old, accounted for Holocene Climatic Optimum. Calculation on the entire range of parameters will be made in the course of further studies.

In response to objections to the treatment of labyrinth calendar can cause many serious arguments, but most convincing is a visual representation of schemes year shadows (Figure 5).

With his artistic interpretation can be obtained: Labryssided hatchet antlered god of light and sun cancer, or many-armed Shiva (six major areas correspond to the equinoxes and solstices), the lotus flower as a symbol of the year- "the day the gods" that lasts one year and the chariot of the god of light, where the axle connects the two equinoxes cycle-a cycle of rising in the east, a series of visits to the west. Given the gradual shift of the extreme northern and southern culmination of these cycles, we can speak of two spirals, symbolizing the past (west) and future (east).

\section{Models of Natural Processes: Birth and Evolution}

According to existing ideas, simple forms of signs are older. This corresponds to the essence of development from simple to complex. Simultaneous development of recording technologies and the increase of transmitted information content in primitive art are studied well. In this respect, notables are the fundamental works by B. A. Frolov, V. E. Larichev, L. S. Marsadolov for their in-depth and many-sided analysis of reflection of knowledge of natural processes and phenomena in graphics and architecture of ancient objects. The results of our research of northern labyrinths are generally consistent with the conclusions of these authors, and allow us to formulate some new provisions. 

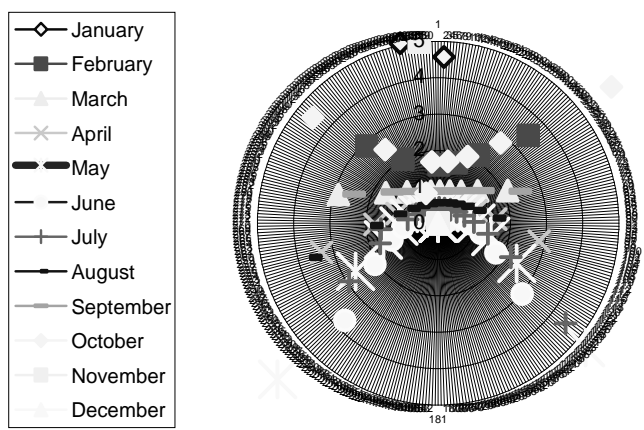

Figure 5. The collection of the gnomon shadow this year is in the form Labrys.

First, it is important that the simplicity of ancient signs was not the result of a synthesis of information, encoding the preceding forms in a more concise form. The high degree of generalization inherent in the sign initially and meets its primary function-a reflection of nature, in one of her typical vital manifestations, especially this relates to processes and phenomena, not objects. Accordingly, the sign is a graphic model of the natural process. Among the processes associated with orientation: mode of lighting, dynamic picture of the sky, the lunar cycle, but this manner may encode and others-physical, chemical, and biological processes. The main requirement for the iconic image-clear enough sketchiness transfer of key moments, plays the role of the starting link in the causal relationship. These criteria are the definition of "sign" and provide its initial allocation of the entire array of information captured by painting. The results of our research gnomon showed that typical, repetitive geometry of daily and annual shadows become the real basis of the arsenal of symbolic systems, which we now use. If a simple sign could not emerge from the artistic image, the reverse process has many examples that reflect different stages of degradation of its primary navigational content. Preserve the traditional structure of the mark, without reference to a prisoner in it the knowledge leads to filling the structure with new meaning, the degree of positiveness of which depends on the settings of the era, "political moment" and individual choice (the artist, the customer) in full conformity with the proven thesis that that the shape of the object is more conservative than the content. This process can be regarded as an example of entropy, which is known to cause structural complications in the immediate vicinity. So, without violating the canons of the spatial organization of its basic elements, there are details: leaves, small animals, horns, woven in a mythological story. In this case, are more commonly used objects associated with the transfer of the contents of a particular mark by disclosing its contents myth, where myth-the story-shaped model of the natural process. It would seem that a combination of complementary models, the double embedding energy should strengthen the substantive basis, but the increases appear to preserve the form, as works of art: to enrich the sign of the mythological characters of the model, a more complex picture, using a broad arsenal of means of expression in the amount of overlap rational content sign framework. At the beginning of this stage of life sign, its interpretation is well known, but is gradually becoming a more generalized and abstract nature, later, the uninitiated viewer evaluates only the aesthetic value of a particular image. To isolate the primary meaning of the sign involved variations and natural sites, allowing complete visual and conceptual range. Objective complexity of this algorithm, the study is that process in the suit included specialists in the main humanities, far from the fundamental principle of natural signs and does not operate processes and objects and their images are not models of nature, as "models of models". In this case, the artificial world of humanitarian information environment arguments to explain a model serves other models and their consistency is enough. Since the signs of culture are filled with new content, and the company receives information about a person prehistoric past, which is hard to understand.

We can construct an evolutionary series for the sign of labyrinth, which will include some controversial interpretations. Main stages and directions of the labyrinth and its symbols: 1) Tool orientation in space-time: a compass, clock, calendar; 2) Directional sign of the sun and the motherland-for carriers of the culture of northern maritime civilization; 3) "Calling card" of northerners, a brand that territory, difficult for the less experienced people to navigate; 4) A sign of strength, success, fertility, wealth, harmony, with the culmination of Paradise in the heart; 5) Object geometric styling and scholastic interpretation, the search for the lost in thought up blind alleys of labyrinths, the center-a symbol of rebirth; 6) Finding a maze and their copies of the mass of useful features-from entertainment and satellite communications sessions "to harmonize mind and treat serious illnesses"; 7) The key to understanding the ancient history of Northern Europe, unity of Eurasia and the sources of the formation of the noosphere.

Note that if a compass and a sundial can serve almost any count, a spear, staff, scepter, and the like, a labyrinth in the first place, a calendar-a landmark in Time, Eternity, and therefore a symbol of life, fate, Renaissance. All other treatments (fertility, prosperity, success, change in age and social status) are secondary, they expand and explain the original concept, the essence of which led to a wide geography and universal popularity of the sign.

\section{Orientation Tools and Social-Cultural Paradigm}

Process of orientation by sunrise/sunset, day/night stars formed binary concepts (yes/no, day/night, light/shadow), 
reflecting the idea of struggle and unique properties of opposites, which was appreciated in ancient times. Broad use of direct sight left many traces in the sacred monuments, various systems of notation (toponyms, linguistic, semiotic), and universal binary organization of the World continues fruitfully be applied to the modern scientific paradigm (binary code). The introduction of the inverse of sight-in the shadow of a vertical object, made a significant change in the paradigm of thinking in the fullest sense of the word "revolution in the mind": shadows of objects at the time of sunset fall on the west, and during his call-to the east, moving day "clockwise". With the approval of the sign-shadow head rotation in the images of the solar falcon was reoriented to the west, where calendar information on the time and its culmination, from direction recording marks.

Gnomon, which enabled to measure the instrumental middle of the day, carried out a revolution in the measurement system and encoding information about space/ timecode (numbers) and became the prototype of the most significant mytho-poetical images: Mount Meru, the World Tree, and the very notions of "Peace" and "Light". Rays converging to the vertex of the gnomonthe images of branches and shadows, diverging from the base - the symbols of the roots, although the mythology knows, and "inverted" examples: in the hands of Varuna World Tree roots absorb the information and energy of the cosmos, and the branches and fruit are lowered to the Earth. In such a model of the world can see the embodiment of causal relations the system "Nature-Knowledge". To navigate through (d) gnomon (a) gave rise to the universal principle of "dying to be born", describing the three main pillars of the Sun (sunset, sunrise, and the climax), the methodology of "reflection of the cycle and" have not lost their freedom, its meaning, and M-concepts: large-scale as a "mega", divinely wise as Mitra, Medea, Melpomene, sacred as Mecca, the central on-Media, Meotida, which is probably also ensured the sanctity, and the chain of terms associated with the measurement, the markers including different color, like chalk (white) and melanin (black). One of the consequences of such thinking was changing socio-political setting - from the "unity of significant parts", agreed with the direct sight, with the help of the established range of subjects, the idea of "leading center", reproduces the generating and preserving vital information about the procedure. Finally, attention-attention to the sign (the geometry of shadows), diverted attention from the natural process, became the basis for modeling the higher levels, has led to the suppression of environmentally-mentality, forming the illusion of emancipation from Nature.

\section{Noosphere Concept of the World Information Model}

Culture is the result of human exploration of the world's
Nature House and its surroundings, where masteringunusual for the analysis and synthesis characteristics. For adequate public information model of the World (IMW) is a result of adaptation, the foundation of stability and a guarantee of its preservation. In the humanities the primary modeling system of interaction between man and nature are often made on the basis of intersecting circles (spheres) and discuss areas of overlap. Such approach is fundamentally wrong: the role of human is hypertrophied, its natural essence is ignored, that largely determines the level of misunderstanding of contemporary issues. More opportunities for an adequate model of the world gives the noosphere concept, V.I. Vernadsky laid the foundation of most complex disciplines of environmental trends. Here, the "noosphere"- the sphere of intelligence, information, socio-natural self-organization. In a study of the processes of reflection of the geographical space in the overall IMW, we proceed from the spherical (shell) structure of geographical space and adjacent space environment. At its core-a system of nested spheres, "SpaceEarth-Man", which correspond to the space, land and culture, which differ in mass, age (time) and complexity (information). Human interaction with the environment is represented by continuous. Constructive roles are determined an objective hierarchy of systems that do not contradict the thesis on the geological scale of living matter, including man, in the geographical space. In the lateral dimension noosphere can be considered as a set of geocultural and ethno-cultural spaces, subordinate to reach areas. In the geo-cultural space is usually considered material and energy components: the economy, resources, natural and anthropogenic landscape. Analysis of the informational aspects of its structure covers mainly the statistical data from census to environmental management. Issues of national culture and history, as the information that ensures the stability of regional systems, neglected, and in the transition, periods of crisis, have been practiced even rewriting of its pages (as it was with the Chronicle in the past or the history books - in the early twentieth century and now) and deliberate distortion. The justification for this is the present political situation, and the words of A. Pushkin: "A nonentity despises the past and reverence for this”.

In recent decades, the science has been enriched by innovative work in cultural geography, historical geography and semiotics [5,6]. Our studies of cultural heritage of the Stone Age and sacred landscapes of different regions of Russia showed their relationship with a vital reference points of time and space, the inclusion of a single information system [3,7], where invariant structures reflect the characteristics of common global processes, and variations-regional differences geographical environment. The basis of modeling Geosystems - the parameters of geographic space, defines the set and mode 
of natural processes, internal structure, external relations (the border, the neighborhood, the inclusion of a system of hierarchical relationships), and other factors. The earliest model of the spatial-temporal organization of developed geographical systems are allocated directly to the natural environment (nature monuments) or copies that are stored in material objects (monuments of culture) in the images and concepts that make up the contents of IMW. In our study, the concept of IMW is revealed through her relationship different blocks, among which: navigation, linguistic, toponymic, semiotic, cartography, mythology, sacred. IMW reflects the structure of nature in the logic of its consistent development, distinguished of units based on functional differences. The role of the base, a frame element in the content of the general model performs navigation framework, consisting of objects and concepts associated with the movement and orientation in space-time. The function encode (duplication and replication) perform linguistic and other models based on content-based navigation. Key position in the information model of the world belongs to the system of sacred sites, preserving the ancient knowledge and traditions necessary to maintain the continuity of life on Earth.

Systematic organization of nature is reflected in the hierarchy of the noosphere, where space is allocated dominant and subdominant in the regional space of the Earth. Since the geographical area of about $99 \%$ of processes based on solar energy - the entire culture in one way or another connected with the Sun. The result of our research information processes in geographic space-the representation of the navigation flow (mainly solar) information, organizes the structure of information model of the world and its units, subsystems. In all the variety of environmental factors, solar energy is dominated by its effect on the processes occurring in the geographical space, it flows connect all the objects into a single global system. Multifaceted reflection of the natural processes in the mind-a necessary condition for survival. Solar Culture-part of human experience, which reflects the interaction with the sun in all its manifestations: the realenergy and information. Widespread use and the occurrence of fundamental rights is a fundamental principle of IMW solar navigation easy to understand if you imagine the time and place of its activism. The main objects of interest in the ancient human, like himself, were reversed in the environment and differ primarily in the "address", on the situation in space, the phase of life and annual cycle. Preservation of solar data is not only a universal concept, but also the rationality of an ancient culture, diversity is not burdened with unnecessary items. Comparison of individual blocks of knowledge, such as science and humanities, shows that models II, III, $\cdots$ order, which we call "models of models", as the distance from the natural bases are characterized by loss of accuracy, and in the process of expansion "tree" model leads to create an artificial—organic, but a limited "world of illusion”. For making the ideological paradigm and solving the accumulated problems in the humanities should be based science.

Obviously, the diagnosis of loss of sacred objects and signs of primary meaning, and his search should be based on the criterion due to the nature and function of collective livelihoods. We see that the transformation-the most dramatic, qualitative changes in relation to nature and presentation of knowledge that occur at the change of the scientific paradigm, spiritual values and political influence. Prerequisites fracture outlook at the turn of our era has become, apparently, the deification of the mark and the knowledge of human hypertrophic ego Iron Age. Of the absolute content of the sacral IMW (words) grew philosophy of Christianity, which asserts unnatural ideals of Earth prepare for eternity, which led to the loss of value of life itself. Today, the current level of "spirituality" is reinforced by the successes of cloning.

In the signs associated with a solar orientation, the main content is the ratio of light and shadows that are always together. Pure light banishes darkness, but as an absolute truth, a beacon, beckoning into infinity. The shadow gives the images depth and forms a mark, to determine the actual boundaries, but its concentration is gradually eroding, erases them. In pure form, everything is lifeless extremes, and therefore exists only in theoretical consciousness. Pure art, or art for "art's sake"-lack of spirituality, as an example, recall the "Black Square" by Malevich. In the form of the same frank call develops the whole mass culture of modernity, "adequately" represented by the media, at the same level fall exhibition halls, open-tries the door dubious art. In our opinion, these distortions - the model of the artificial world, once the mask is in our time, become virtual reality.

\section{Conclusions}

1) Position in space-time is a universal characteristic of any object, and the primary basis of all symbolic systems.

2) Universal criterion of the true value of culturepositive attitude, reflecting the effects of the sun on all aspects of life, as reflected in the ancient solar cult and stored in the solar culture noosphere.

3) V.I. Vernadsky's noosphere concept allows us to rethink the artificial world and connect them with modern scientific understanding of natural processes of nature, accents, in accordance with the long-term evaluation of the structural parameters (spatial and temporal organization) at-home systems, which defines its role as a necessary basis for development of modern-variable progressive socio-cultural paradigm. 
4) The navigation concept information modeling nature opens up new interdisciplinary (with archeology, ethnography, linguistics, semiotics) directions of research in the field of geography culture.

5) If "everything is back to square one" and this first hand can be under the supervision of sunlight in the maze, then on the next orbit planetary-cosmic rhythm Humans have to find ways to more careful treatment of Nature.

\section{REFERENCES}

[1] G. Paranina and R. Paranin, “The Labyrinth: Orientation in Geographic Space and the Evolution of the Mark. Space Geocultural European North: The Genesis, Structure and Semantics. Materials IV Pomeranian Readings on the Semiotics of Culture, 7-11 July 2009," Pomorski University, Pinezhsky, 2009, pp. 516-518.

[2] G. Paranina and R. Paranin, "Northern Mazes as Astro- nomical Instruments in Relation to Patterns of Mythology and Symbols of Culture," Society, Environment and Development, St. Petersburg, Vol. 4, No. 14, 2009, pp. 120134.

[3] G. Paranina, "Light in the Labyrinth: Time, Space, Information,” Asterion, St. Petersburg, 2010, p. 123.

[4] A. Skvortsov, "How Many Monuments Are There in Solovki?” The Study of IP-Torik-Cultural Environment of the Arctic, 1990, pp. 282-300.

[5] V. Paranin, "Historical Geography of the Chronicle of Russia,” Petrozavodsk, 1990, p. 152.

[6] V. Paranin, “The History of the Barbarians," RGO, St. Petersburg, 1998, p. 184.

[7] G. Paranina, "Reflection of Time and Space in Ancient Symbols (for Example, the Sign of Rurik)," Society, Environment and Development, Asterion, St. Petersburg, Vol. 2, No. 16, 2010, pp. 199-207. 